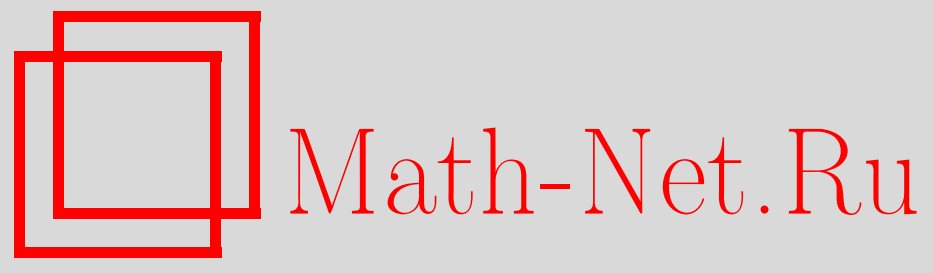

Р. В. Ардазишвили, М. В. Вильде, Л. Ю. Коссович, Трехмерная поверхностная волна в полупространстве и кромочные волны в пластинах в случае смешанных граничных условий на поверхности распространения, Вестн. Сам. гос. техн. ун-та. Сер. Физ.-мат. науки, 2014, выпуск 4(), 53-64

DOI: https://doi.org/10.14498/vsgtu1360

Использование Общероссийского математического портала MathNet.Ru подразумевает, что вы прочитали и согласны с пользовательским соглашением

http: //www . mathnet.ru/rus/agreement

Параметры загрузки:

IP: 35.173 .219 .12

26 апреля 2023 г., 18:23:03

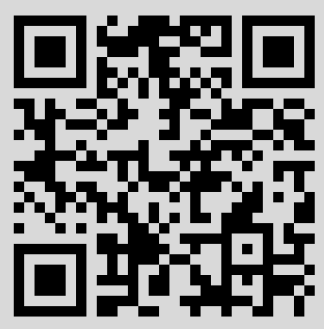


Вестн. Сам. гос. техн. ун-та. Сер. Физ.-мат. науки. 2014. № 4 (37). С. $53-64$

ISSN: 2310-7081 (online), 1991-8615 (print) doi: http://dx.doi.org/10.14498/vsgtu1360

УДК 539.3:534.121.1

\title{
ТРЕХМЕРНАЯ ПОВЕРХНОСТНАЯ ВОЛНА В ПОЛУПРОСТРАНСТВЕ И КРОМОЧНЫЕ ВОЛНЫ В ПЛАСТИНАХ В СЛУЧАЕ СМЕШАННЫХ ГРАНИЧНЫХ УСЛОВИЙ НА ПОВЕРХНОСТИ РАСПРОСТРАНЕНИЯ
}

\author{
Р. В. Ардазишвили, М. В. Вилъде, Л. Ю. Коссович \\ Саратовский государственный университет им. Н. Г. Чернышевского \\ (национальный исследовательский университет), \\ Россия, 410012, Саратов, ул. Астраханская, 83.
}

\begin{abstract}
Аннотация
Исследуются поверхностные волны в полупространстве в случае смешанных граничных условий на поверхности, а также волны, распространяющиеся вдоль кромки пластины (кромочные волны), при смешанных граничных условиях на кромке. В случае полупространства рассматривается гармоническая волна, распространяющаяся в произвольном направлении вдоль поверхности и затухающая при удалении от нее. Поверхность полупространства считается закрепленной в одном из тангенциальных направлений и свободной в остальных направлениях. Получено точное дисперсионное уравнение, показывающее, что при данных граничных условиях существует трехмерная поверхностная волна, скорость которой изменяется в зависимости от угла распространения от скорости волны сдвига до скорости волны Рэлея. Приведены графики зависимости скорости волны от угла распространения. Во второй части работы рассматриваются симметричные и антисимметричные кромочные волны в пластине, лицевые поверхности которой свободны от напряжений. Торец пластины считается закрепленным в одном из тангенциальных направлений и свободным в остальных направлениях. Для описания колебаний пластины применяются трехмерные уравнения теории упругости. Построены асимптотики для больших значений волнового числа, показывающие, что при данных условиях закрепления в пластине существует бесконечное счетное множество кромочных волн высшего порядка. Данный вывод подтверждается результатами численных расчетов, в которых использован метод разложения по модам. Численные расчеты показали также наличие фундаментальной волны в случае симметричных колебаний пластины, торец которой закреплен в направлении, перпендикулярном лицевым поверхностям. С увеличением волнового числа
\end{abstract}

(C) 2014 Самарский государственный технический университет.

\section{Образец для цитирования}

А р даз и ш в и л и Р. В., В и ль де М. В., К о с с о в и ч Л. Ю. Трехмерная поверхностная волна в полупространстве и кромочные волны в пластинах в случае смешанных граничных условий на поверхности распространения // Вестн. Сам. гос. техн. ун-та. Сер. Физ.-мат. науки, 2014. № 4 (37). C. 53-64. doi: 10.14498/vsgtu1360.

\section{Сведения об авторах}

Роман Вячеславович Ардазишвили (ardazishvili.roman@yandex.ru), аспирант, каф. математической теории упругости и биомеханики.

Мария Владимировна Вилъде (д.ф.-м.н., проф.; mv_wilde@mail.ru; автор, ведущий переписку), профессор, каф. математической теории упругости и биомеханики.

Леонид Юрьевич Коссович (д.ф.-м.н., проф.; president@sgu.ru), заведующий кафедрой, каф. математической теории упругости и биомеханики; президент Саратовского государственного университета имени Н. Г. Чернышевского. 
скорость этой волны стремится к некоторому предельному значению, зависящему от коэффициента Пуассона. В антисимметричном случае обнаружена волна высшего порядка, имеющая то же предельное значение, что и фундаментальная волна в симметричном случае. Приведены графики зависимости скорости этих волн от волнового числа для различных значений коэффициента Пуассона. Для остальных волн высшего порядка представлены результаты сравнения асимптотического и численного решений.

Ключевые слова: поверхностные волны, кромочные волны, волна Рэлея, волна сдвига, асимптотические методы.

doi: http://dx.doi.org/10.14498/vsgtu1360

Введение. Существование волн, распространяющихся вдоль поверхности полупространства и экспоненциально затухающих при удалении от нее (поверхностные волны), было впервые установлено Рэлеем в 1885 г. [1]. Впоследствии было обнаружено множество других типов поверхностных волн (см., например, обзор [2] и недавние работы [3-8]), а также волны, аналогичные волне Рэлея и распространяющиеся вдоль кромки пластины или оболочки [916]. Как правило, во всех случаях, когда изучаются поверхностные волны в однородном теле, на поверхности ставятся условия свободного края. В данной работе показано, что трехмерная поверхностная волна в изотропном полупространстве может существовать не только в случае свободной от закреплений поверхности, но и в случае, когда на поверхности запрещено перемещение в одном из тангенциальных направлений. Этот факт отмечен также в работе [17], где задача решается другим методом. В отличие от случая свободной поверхности, скорость трехмерной поверхностной волны в случае смешанных граничных условий на поверхности зависит от направления распространения волны. Графики указанной зависимости приведены в данной работе.

Наличие поверхностной волны в полупространстве со смешанными граничными условиями на поверхности указывает на возможность существования кромочных волн в пластинах со смешанными граничными условиями на торце. В данной работе изучаются кромочные волны в пластине со свободными лицевыми поверхностями в случае, когда на торце запрещено перемещение в одном из тангенциальных направлений. При этом рассматривается не только первая волна, аналогичная волнам, описываемым приближенными двумерными теориями пластин, но и волны высшего порядка, которые можно описать только исходя из трехмерных уравнений теории упругости. Для случая условий свободного края на торце пластины волны высшего порядка изучены в работах [18-20].

\section{1. Трехмерная поверхностная волна в полупространстве в случае смешан-} ных граничных условий на поверхности. Рассмотрим гармонические колебания полупространства, занимающего в декартовых координатах область $0 \leqslant x<\infty,-\infty<y<\infty,-\infty<z<\infty$. Введем безразмерные переменные по следующим формулам:

$$
\begin{gathered}
x=h \pi^{-1} \widetilde{x}, \quad y=h \pi^{-1} \widetilde{y}, \quad z=h \pi^{-1} \widetilde{z} \\
\left\{u_{x}, u_{y}, u_{z}\right\}=h \pi^{-1}\left\{\widetilde{u}_{x}, \widetilde{u}_{y}, \widetilde{u}_{z}\right\}, \quad \widetilde{\omega}=h \pi^{-1} \omega c_{2}^{-1}, \\
\left\{\sigma_{x}, \sigma_{y}, \sigma_{z}, \sigma_{x y}, \sigma_{x z}, \sigma_{y z}\right\}=E[2(1+\nu)]^{-1}\left\{\widetilde{\sigma}_{x}, \widetilde{\sigma}_{y}, \widetilde{\sigma}_{z}, \widetilde{\sigma}_{x y}, \widetilde{\sigma}_{x z}, \widetilde{\sigma}_{y z}\right\},
\end{gathered}
$$


где $\boldsymbol{u}=\left\{u_{x}, u_{y}, u_{z}\right\}$ - вектор перемещения; $\sigma_{x}, \sigma_{y}, \sigma_{z}, \sigma_{x y}, \sigma_{x z}, \sigma_{y z}$ - компоненты тензора напряжений; $E$ - модуль Юнга; $\nu$-коэффициент Пуассона; $c_{2}$ - скорость волны сдвига; $\omega$ - круговая частота; $h$ - некоторая величина, имеющая размерность длины. Временной множитель $e^{i \omega t}$ и знак « », обозначающий безразмерные переменные, далее опущены. После введения безразмерных переменных по формулам (1) в уравнениях остается единственный параметр - коэффициент Пуассона $\nu$. Таким образом, значения модуля Юнга $E$ на приведенные ниже результаты не влияют. Запишем выражения перемещений через упругие потенциалы Ламе $\varphi$ и $\boldsymbol{\psi}$ :

$$
\boldsymbol{u}=\operatorname{grad} \varphi+\operatorname{rot} \boldsymbol{\psi} .
$$

Выражения напряжений через потенциалы $\varphi$ и $\boldsymbol{\psi}$ приведены в работе [20]. Представление (2) следует дополнить условием $\operatorname{div} \boldsymbol{\psi}=0$, которое является достаточным для получения однозначного представления компонентов вектора перемещения в упругом теле в виде (2). Функции $\varphi$ и $\boldsymbol{\psi}$ должны удовлетворять уравнениям Гельмгольца:

$$
\Delta \varphi+\kappa^{2} \omega^{2} \varphi=0, \quad \Delta \boldsymbol{\psi}+\omega^{2} \boldsymbol{\psi}=0
$$

где $\Delta$ - трехмерный оператор Лапласа, $\kappa=\sqrt{(1-2 \nu) / 2(1-\nu)}$. На поверхности полупространства $x=0$ поставим смешанные граничные условия вида

$$
\begin{array}{ll}
\sigma_{x}=0, & u_{y}=0, \quad \sigma_{x z}=0 ; \\
\sigma_{x}=0, & \sigma_{x y}=0,
\end{array}
$$

Частные решения уравнений (3) ищем в виде

$$
\varphi=C_{1} e^{-r_{1} x} e^{i(\omega t-(\gamma y+s z))}, \quad \boldsymbol{\psi}=\boldsymbol{C} e^{-r_{2} x} e^{i(\omega t-(\gamma y+s z))},
$$

где $\boldsymbol{C}^{\top}=\left\{C_{3}, C_{4}, C_{2}\right\}$ - вектор произвольных констант. Подставляя (6) в (3), найдем $r_{1}, r_{2}$ :

$$
r_{1}=\sqrt{\gamma^{2}+s^{2}-\kappa^{2} \omega^{2}}, \quad r_{2}=\sqrt{\gamma^{2}+s^{2}-\omega^{2}}
$$

Подставляя представления (6) в граничные условия (4), получим систему однородных линейных уравнений относительно постоянных $C_{1}, C_{2}, C_{3}, C_{4}$. Условие существования нетривиального решения этой системы приводит к дисперсионному уравнению

$$
\begin{aligned}
\left\{\left(2 s^{2}+2 \gamma^{2}-\omega^{2}\right)^{2}-4 r_{1} r_{2} s^{2}\right\}\left(\omega^{2}-\gamma^{2}\right)- & \\
- & \left(\gamma^{2}+s^{2}-\omega^{2}\right)\left(6 s^{2} \gamma^{2}+4 \gamma^{4}-4 \gamma^{2} \omega^{2}\right)+2 r_{1} r_{2} \gamma^{2} s^{2}=0 .
\end{aligned}
$$

Введем параметр $\alpha$, характеризующий направление распространения волны и связанный с параметрами $s$ и $\gamma$ соотношениями

$$
s=\gamma_{1} \sin \alpha, \quad \gamma=\gamma_{1} \cos \alpha
$$

где $\gamma_{1}=\sqrt{s^{2}+\gamma^{2}}$. Тогда дисперсионное уравнение $(7)$ запишется в виде

$$
(2-\vartheta)\left(2 \sin ^{2} \alpha-\vartheta\right)+\vartheta \cos ^{2} \alpha-4 \widetilde{r_{1}} \widetilde{r_{2}} \sin ^{2} \alpha=0
$$


где $\widetilde{r_{1}}=\sqrt{1-\kappa^{2} \vartheta}, \widetilde{r_{2}}=\sqrt{1-\vartheta}, \vartheta=c^{2}, c=\omega / \gamma_{1}-$ фазовая скорость трехмерной поверхностной волны, отнесенная к скорости волны сдвига.

Покажем, что уравнение (8) имеет единственный корень, которому соответствует нетривиальное решение системы. Рассмотрим функцию:

$$
\begin{gathered}
D(\vartheta, \kappa, \alpha)=(2-\vartheta)\left(2 \sin ^{2} \alpha-\vartheta\right)+\vartheta \cos ^{2} \alpha-4 \widetilde{r_{1}} \widetilde{r_{2}} \sin ^{2} \alpha \\
D_{\vartheta}^{\prime}(\vartheta, \kappa, \alpha)=-2-2 \sin ^{2} \alpha+2 \vartheta+\frac{(1-\vartheta) \kappa^{2}+\left(1-\kappa^{2} \vartheta\right)}{\sqrt{(1-\vartheta)\left(1-\kappa^{2} \vartheta\right)}} \\
D_{\vartheta}^{\prime \prime}(\vartheta, \kappa, \alpha)=2+\sin ^{2} \alpha \frac{(\kappa-1)^{2}(\kappa+1)^{2}}{\left(1-\kappa^{2} \vartheta\right)^{3 / 2}(1-\vartheta)^{3 / 2}} .
\end{gathered}
$$

Воспользуемся достаточным условием вогнутости функции. На интервале $(0 ; 1) D_{\vartheta}^{\prime \prime}(\vartheta, \kappa, \alpha)>0$, следовательно, уравнение (8) может иметь не более двух корней на данном интервале. Из формул $(9),(10)$ имеем $D(0, \kappa, \alpha)=0$, $D_{\vartheta}^{\prime}(\vartheta, \kappa, \alpha)<0$. С другой стороны, $D(1, \kappa, \alpha)>0 \forall \alpha \in(0 ; \pi / 2]$.

Следовательно, на интервале $(0 ; 1)$ график функции один раз пересечет ось абсцисс. Таким образом, уравнение (8) имеет единственный корень, не равный нулю. Проведенный анализ показывает, что искомая волна существует при всех значениях $\alpha$, кроме $\alpha=0$.

Граничные условия (5) аналогичны граничным условиям (4) с точностью до поворота осей координат. В уравнении (8) следует выполнить замену $\sin \rightarrow \cos$ и $\cos \rightarrow \sin$. Если на поверхности полупространства ставятся условия свободного края, то скорость трехмерной поверхностной волны не зависит от направления распространения и совпадает со скоростью волны Рэлея. Анализ, аналогичный описанному выше, показал, что в случае других видов закреплений на поверхности, отличных от (4) и (5), трехмерная поверхностная волна не существует.

Зависимость параметра $\vartheta$ от угла распространения, полученная в результате численного решения уравнения (8), представлена на рис. 1, а для различных значений коэффициента Пуассона. Значения этого параметра при $\alpha=0.5 \pi$ совпадают с квадратом скорости волны Рэлея при данном значении $\nu$. На рис. 1, б приведены графики изменения перемещений в поверхностной волне по переменной $x$. Перемещения вычислены по формулам (2) после определения констант $C_{1}, C_{2}, C_{3}, C_{4}$ с точностью до произвольного постоянного множителя и нормированы на значение перемещения $u_{x}$ при $x=0$.

2. Кромочные волны в пластинах: асимптотический анализ. Рассмотрим гармонические колебания упругой пластины, занимающей в размерных декартовых координатах $(x, y, z)$ область $0 \leqslant x<\infty,-h \leqslant y \leqslant h,-\infty<z<\infty$. Для описания колебаний пластины применим трехмерные уравнения теории упругости. Введем безразмерные переменные по формулам (1), где $h$ обозначает теперь полутолщину пластины. Знак « », обозначающий безразмерные переменные, далее опустим. Уравнения для потенциалов $\varphi$ и $\boldsymbol{\psi}$, а также выражения для перемещений и напряжений через них будут выглядеть так же, как и в случае полупространства. На лицевых поверхностях $y= \pm \pi$ ставятся граничные условия свободного края

$$
\sigma_{y}=\sigma_{x y}=\sigma_{y z}=0
$$



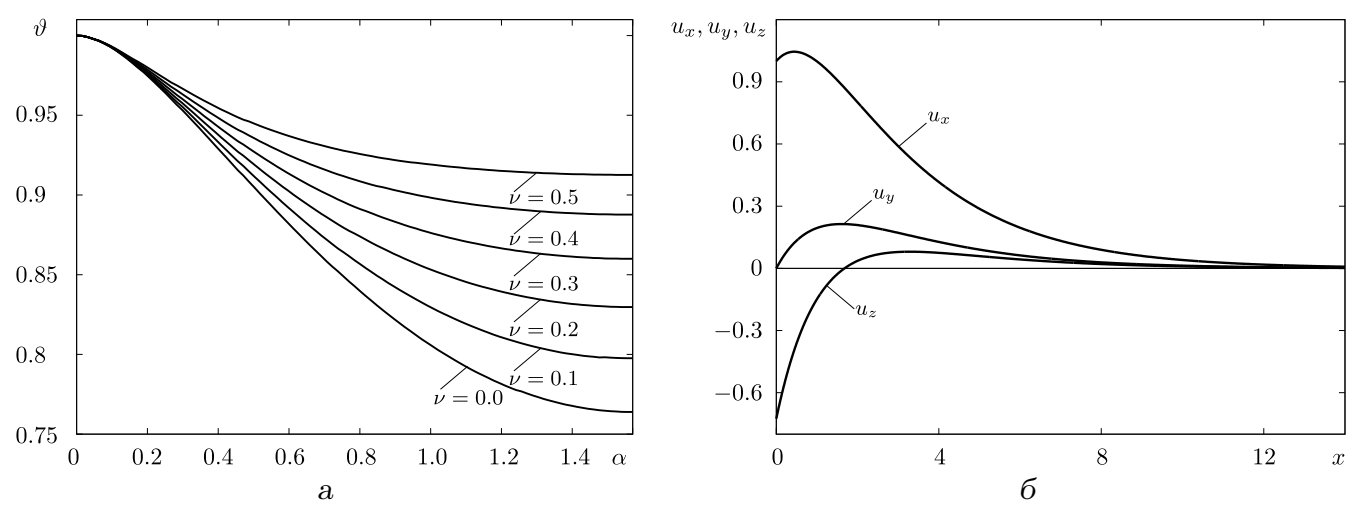

Рис. 1. График зависимости фазовой скорости трехмерной поверхностной волны от угла распространения для различных значений коэффициента Пуассона (а) и форма данной волны (б) при $\alpha=\pi / 3$ и $\nu=0.25$

Будем изучать гармонические кромочные волны, распространяющиеся вдоль кромки пластины $x=0$ в направлении оси $z$. Для удобства представим искомое решение в виде стоячей волны, то есть будем считать, что напряженнодеформированное состояние изменяется по переменной $z$ по гармоническому закону. При $x=0$ поставим одно из следующих граничных условий:

$$
\begin{aligned}
& \sigma_{x}=T_{g}(y) \cos s z, \quad u_{y}=0, \quad \sigma_{x z}=0, \\
& \sigma_{x}=T_{g}(y) \cos s z, \quad \sigma_{x y}=0, \quad u_{z}=0 \text {, }
\end{aligned}
$$

где $T_{g}(y)$ - заданная функция переменной $y, s$ - некоторое число. Резонансные частоты рассматриваемой задачи соответствуют собственным частотам однородной задачи с граничными условиями (4) и (5) при $x=0$ соответственно, при этом следует искать те решения однородной задачи, которые соответствуют принятому закону изменения НДС по переменной $z$ и затухают при $x \rightarrow \infty$. На бесконечности ставится условие отсутствия источников энергии.

Решение однородной задачи (3), (11) можно представить в виде суммы двух кромочных волн, распространяющихся в противоположных направлениях вдоль оси z. Фазовая скорость кромочной волны определяется по формуле

$$
c=\omega_{0} / s
$$

где $\omega_{0}$ - собственная частота задачи $(3),(11),(4)$ или (5) или резонансная частота задачи (3), (11), (12) или (13).

Рассмотрим однородную краевую задачу (3), (11), (4). Примем, что значение параметра $\gamma$ фиксировано, и запишем асимптотики при $s \rightarrow \infty$ для напряжений, входящих в граничные условия (11) в антисимметричном случае:

$$
\sigma_{y}^{R}=C\left\{\left[2 s^{2}\left(1-\frac{\theta_{1}^{2}}{\theta^{2}}\left(1+O\left(\frac{\gamma^{2}}{s^{2}}\right)\right)\right)-\omega^{2}\right] e^{-s \tilde{r}_{1}^{R} x}+2 \gamma^{2} e^{-s \tilde{r}_{2}^{R} x}\right\} \times
$$

$$
\times \sin (\gamma y) \cos (s z)
$$




$$
\begin{aligned}
\sigma_{x y}^{R}=C \frac{\gamma s}{2}\left\{-4 \frac{\theta_{1}}{\theta} e^{-s \tilde{r}_{1}^{R} x}+\left[3 \frac{\theta_{2}}{\theta}+\frac{\theta}{\theta_{2}}\left(1+\frac{\gamma^{2}}{s^{2}}\right) e^{-s \tilde{r}_{2}^{R} x}\right]\right\} \times \\
\times\left(1+O\left(\frac{\gamma^{2}}{s^{2}}\right)\right) \cos (\gamma y) \cos (s z), \quad(14) \\
\sigma_{y z}^{R}=C \frac{\gamma s}{2}\left\{-4 e^{-s \tilde{r}_{1}^{R} x}+\left[3-\frac{\gamma^{2}}{s^{2}}+\frac{\theta_{2}^{2}}{\theta^{2}}\left(1+O\left(\frac{\gamma^{2}}{s^{2}}\right)\right)\right] e^{-s \tilde{r}_{2}^{R} x}\right\} \times \\
\times \cos (\gamma y) \sin (s z),
\end{aligned}
$$

где

$$
\begin{gathered}
\tilde{r}_{1}^{R}=\frac{\theta_{1}}{\theta} \sqrt{1+\frac{\gamma^{2}}{s^{2}}}, \quad \tilde{r}_{2}^{R}=\frac{\theta_{2}}{\theta} \sqrt{1+\frac{\gamma^{2}}{s^{2}}}, \\
\theta_{1}=\sqrt{\theta^{2}-\kappa^{2}}, \quad \theta_{2}=\sqrt{\theta^{2}-1}, \quad \theta=\frac{1}{\sqrt{\vartheta}},
\end{gathered}
$$

$\vartheta$ - корень дисперсионного уравнения (8). В симметричном случае в формулах (14) следует выполнить замену $\sin \longrightarrow \cos , \cos \longrightarrow \sin , \gamma \longrightarrow-\gamma$. Формулы (14) показывают, что при $s \rightarrow \infty$ наибольшим из напряжений, входящих в граничные условия на лицевых поверхностях, является напряжение $\sigma_{y}^{R}$. Чтобы удовлетворить всем граничным условиям однородной задачи с асимптотически малой погрешностью, достаточно положить $\gamma=n, n=1,2, \ldots$ в антисимметричном случае и $\gamma=n+0.5, n=0,1, \ldots$ в симметричном случае.

Таким образом, в качестве первого приближения собственной формы колебаний рассматриваемой пластины можно принять форму трехмерной волны Рэлея. Подставляя (14) в выражение $\gamma_{1}=\theta \omega$, связывающее частоту и волновое число в поверхностной волне, получим асимптотику собственных частот при $s \rightarrow \infty$ :

$$
\omega_{n}^{(\infty)}= \begin{cases}\omega_{n}^{0} & \text { в антисимметричном случае, } \\ \omega_{n+0.5}^{0} & \text { в симметричном случае, }\end{cases}
$$

где $\omega_{j}^{0}=\sqrt{s^{2}+j^{2}} / \theta$. Формула (15) показывает, что в рассматриваемой пластине существует бесконечное счетное множество кромочных волн высшего порядка. Получим асимптотику фазовых скоростей кромочных волн высшего порядка при $s \rightarrow \infty$ :

$$
c_{n}^{(\infty)}= \begin{cases}\sqrt{1+n^{2} / s^{2}} / \theta & \text { в антисимметричном случае } \\ \sqrt{1+(n+0.5)^{2} / s^{2}} / \theta & \text { в симметричном случае. }\end{cases}
$$

Из (8) следует, что если $s \rightarrow \infty$, то $\theta \rightarrow 1 / c_{R}$, следовательно, фазовые скорости кромочных волн высшего порядка в случае граничных условий (4) стремятся к скорости волны Рэлея.

Рассмотрим однородную краевую задачу (3), (11), (5). Аналогично случаю с граничными условиями (4) можно построить асимптотики и удовлетворить граничным условиям (11) с асимптотически малой погрешностью при $s \rightarrow \infty$. 
Асимптотики собственных частот и фазовых скоростей кромочных волн при $s \rightarrow \infty$ имеют вид (15), (16) соответственно с точностью до замены антисимметричного случая на симметричный и наоборот.

Из дисперсионного уравнения при данных граничных условиях следует, что $\theta \rightarrow 1$ при $s \rightarrow \infty$, следовательно, фазовые скорости кромочных волн высшего порядка в случае граничных условий (5) стремятся к скорости волны сдвига.

3. Кромочные волны в случае смешанных граничных условий на торце: численные результаты. Для подтверждения теоретических выводов была проведена серия численных экспериментов. Метод численного решения задачи, основанный на разложении по модам, описан в [20]. С вычислительной точки зрения удобнее исследовать рассматриваемые волны исходя из задачи о вынужденных колебаниях с неоднородными граничными условиями (12) или (13) и используя отмеченное выше соответствие между собственной частотой однородной задачи и резонансной частотой неоднородной задачи. Резонансные частоты определялись численно методом подбора частоты, на которой достигается максимум амплитуды колебаний. Результаты численных расчетов подтверждают проведенный выше асимптотический анализ. В симметричном случае при граничных условиях (4) найдена фундаментальная волна, поведение которой отлично от поведения прочих кромочных волн высшего порядка. Ее фазовая скорость стремится не к скорости волны Рэлея, а к некоторой другой величине, не совпадающей также со скоростью угловой волны в четверти пространства [21]. В антисимметричном случае обнаружена дополнительная волна высшего порядка (ей присвоен номер 0.5), значение фазовой скорости которой в коротковолновом пределе совпадает с соответствующим значением для симметричной фундаментальной волны.

На рис. 2, а представлены графики фазовой скорости симметричной фундаментальной волны при различных коэффициентах Пуассона. На рис. 2, б фазовые скорости половинной кромочной волны высшего порядка в антисимметричном случае (толстые сплошные линии) представлены в сравнении с фазовой скоростью симметричной фундаментальной волны (тонкие сплошные линии) при различных коэффициентах Пуассона.

На рис. 3 приведены графики для $\nu=0.45$ в случае граничных условий (4), на рис. 4 для $\nu=0.25$ в случае граничных условий (5). На них представлены группы графиков, соответствующих каждой найденной волне за исключением половинной, описанной выше. Каждая группа включает три графика, представляющих величину $\delta_{R}=\omega-s / \theta$, где жирным линиям соответствует $\omega=\omega_{k}^{n u m}$ (численное решение), штрихпунктирным линиям - $\omega_{j}^{0}$, а тонким $-\omega_{j+0.5}^{0}$ на рис. 3 , а и $\omega_{j-0.5}^{0}$ на рис. 3 , б и рис. 4 , где $j-$ номер волны, указанный на графиках.

4. Заключение. Асимптотический и численный анализ кромочных волн в пластине показал наличие бесконечного счетного множества кромочных волн высшего порядка. Кромочные волны, как правило, демпфируются распространяющимися модами. Исключение составляет случай фундаментальных волн. Данный факт имеет место и для кромочных волн, исследованных в настоящей работе. Заметим, что в случае свободного торца фазовые скорости волн высшего порядка стремятся к скорости волны Рэлея, а в данной работе показано, что фазовая скорость половинной кромочной волны высшего по- 
рядка в случае закрепления торца стремится к некоторой другой величине, к которой также стремится фазовая скорость симметричной фундаментальной волны.
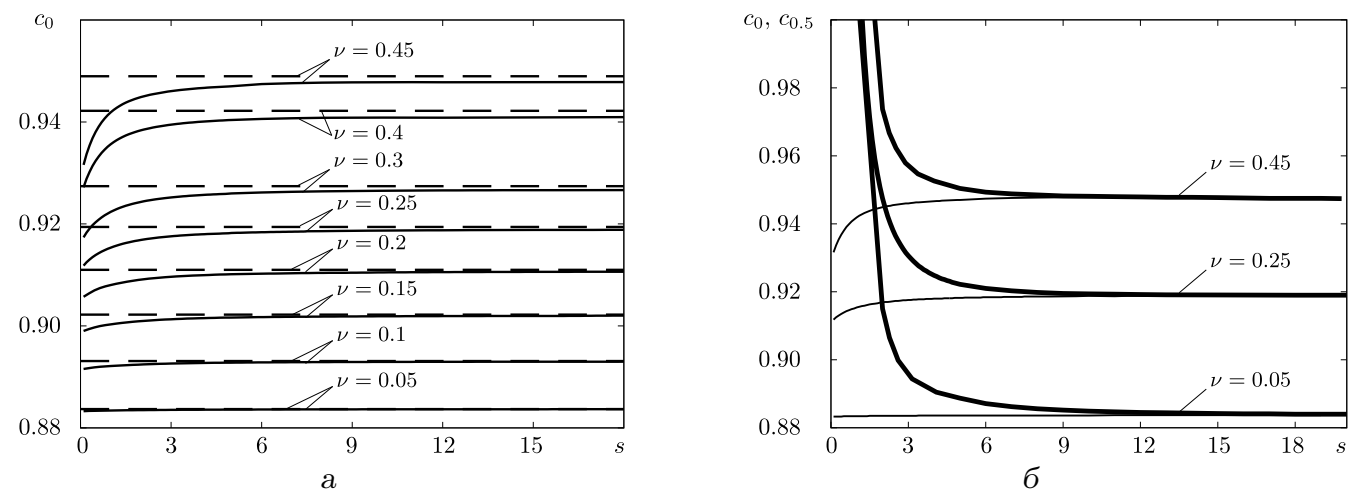

Рис. 2. Фазовые скорости половинной кромочной волны в антисимметричном случае (a) и симметричной фундаментальной волны (б) при различных коэффициентах Пуассона
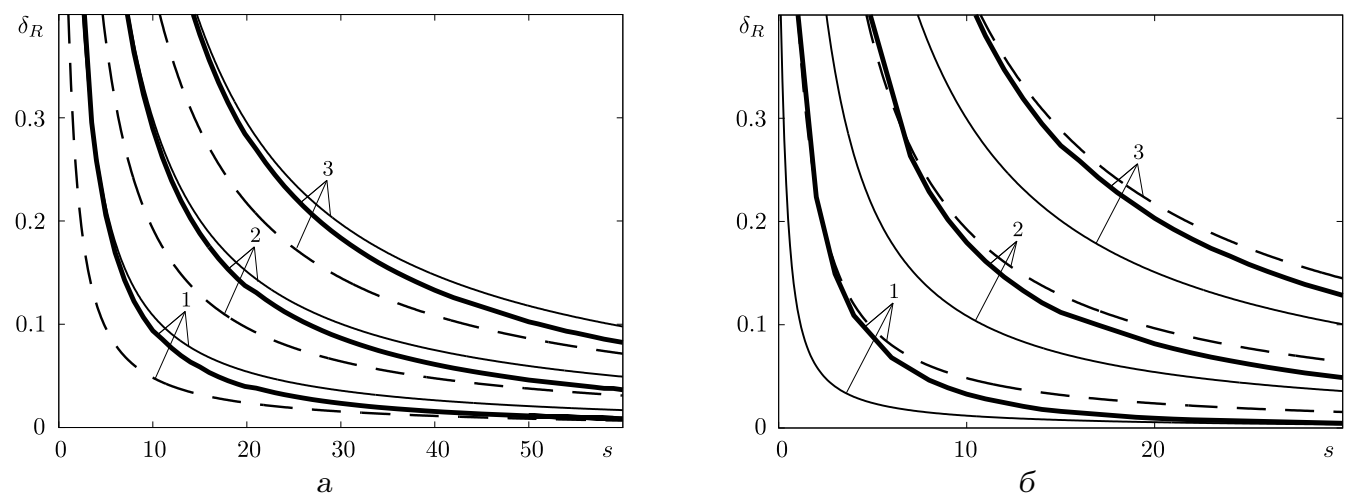

Рис. 3. Результаты расчетов для случая свободных боковых сторон и смешанных граничных условий (4) на торце: а - антисимметричный случай, б - симметричный случай
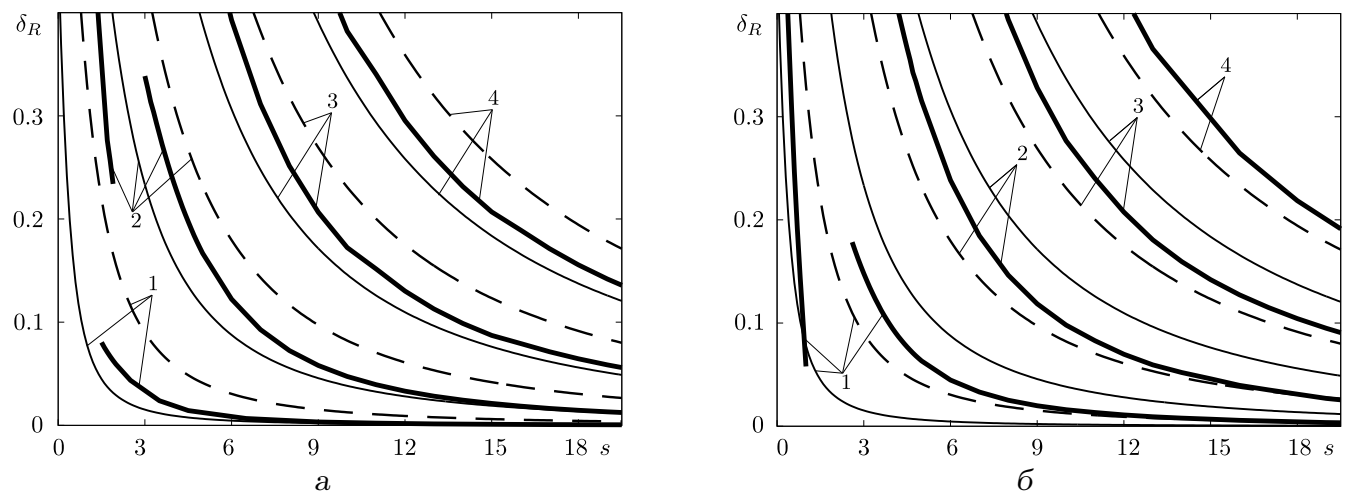

Рис. 4. Результаты расчетов для случая свободных боковых сторон и смешанных граничных условий (5) на торце: а - антисимметричный случай, $\sigma$ - симметричный случай 


\section{ORCID \\ Роман Вячеславович Ардазишвили: http://orcid.org/0000-0002-0993-805X \\ Мария Владимировна Вильде: http://orcid.org/0000-0001-8198-3368 \\ Леонид Юрьевич Коссович: http://orcid.org/0000-0002-4775-7348}

\section{БИБЛИОГРАФИЧЕСКИЙ СПИСОК}

1. Rayleigh J. On waves propagated along the surface of an elastic solid // Proc. Lond. Math. Soc., 1885. vol.s1-17, no. 1. pp. 4-11. doi: 10.1112/plms/s1-17.1.4.

2. Викторов И. А. Типы звуковых поверхностных волн в твердых телах (Обзор) // Акуст. журн., 1979. T. 25, № 1. C. 1-17, http://akzh.phys.msu.ru/pdf/1979_1_1-17.pdf.

3. Destrade M., Scott N. H. Surface waves in a deformed isotropic hyperelastic material subject to an isotropic internal constraint // Wave Motion, 2004. vol. 40, no. 4. pp. 347-357. doi : 10. 1016/j. wavemoti.2003.09.003.

4. Dai H. H., Kaplunov J., Prikazchikov D. A. A long-wave model for the surface elastic wave in a coated half-space // Proc. R. Soc. A, 2010. vol. 466. pp. 3097-3116. doi: 10.1098/rspa. 2010.0125.

5. Eduardo Godoy, Mario Durán, Jean-Claude Nédélec On the existence of surface waves in an elastic half-space with impedance boundary conditions // Wave Motion, 2012. vol. 49, no. 6. pp. 585-594. doi: 10.1016/j.wavemoti.2012.03.005.

6. Stan Chiriţă, Michele Ciarletta, Vincenzo Tibullo Rayleigh Surface Waves on a Kelvin-Voigt Viscoelastic Half-Space// Journal of Elasticity, 2013. vol.115, no.1. pp. 61-76. doi: 10. 1007/s10659-013-9447-0.

7. Inder Singh Gupta Propagation of Rayleigh Waves in a Prestressed Layer over a Prestressed Halfspace// Frontiers in Geotechnical Engineering (FGE), 2013. vol. 2, no.1. pp. 16-22, https://archive.org/details/FGE009.

8. Baljeet Singh Propagation of Rayleigh Wave in a Thermoelastic Solid Half-Space with Microtemperatures // International Journal of Geophysics, 2014. vol. 2014. pp. 1-6. doi : 10. $1155 / 2014 / 474502$.

9. Коненков Ю. К. Об изгибной волне "рэлеевского" типа // Ажуст. журн., 1960. Т. 6, № 1. C. 124-126, http://www.akzh.ru/pdf/1960_1_124-126.pdf.

10. Белубекян М. В., Гулгазарян Г. Р., Саакян А. В. Волны типа Рэлея в полубесконечной круговой замкнутой цилиндрической оболочке // Изв. НАН Армении, Механика, 1997. T. 50, № 3-4. C. 49-55, http://mechanics .asj-oa.am/180.

11. Kaplunov J. D., Kossovich L. Yu., Wilde M. V. Free localized vibrations of a semi-infinite cylindrical shell// J. Acoust. Soc. Am., 2000. vol.107, no. 3. pp. 1383-1393. doi: 10.1121/ 1.428426 .

12. Kaplunov J. D., Wilde M. V. Edge and interfacial vibrations in elastic shells of revolution // ZAMP, 2000. vol. 51, no. 4. pp. 530-549. doi: 10.1007/s000330050015.

13. Fu Y. B., Brookes D. W. Edge waves in asymmetrically laminated plates // Journal of the Mechanics and Physics of Solids, 2006. vol. 54, no.1. pp. 1-21. doi: 10.1016/j.jmps. 2005. 08.007.

14. Piliposian G. T., Belubekyan M. V., Ghazaryan K. B. Localized bending waves in a transversely isotropic plate// Journal of Sound and Vibration, 2010. vol.329, no. 17. pp. 3596-3605. doi: 10.1016/j.jsv.2010.03.019.

15. Krushynska A. A. Flexural edge waves in semi-infinite elastic plates // Journal of Sound and Vibration, 2011. vol.330, no. 9. pp. 1964-1976. doi: 10.1016/j.jsv.2010.11.002.

16. Fu Y. B., Kaplunov J. Analysis of localized edge vibrations of cylindrical shells using the Stroh formalism // Math. Mech. Solids, 2012. vol.17, no.1. pp. 59-66. doi:10.1177/ 1081286511412442.

17. Белубекян В. М., Белубекян М. В. Трехмерная задача распространения поверхностных волн Рэлея // Докл. НАН Армении, 2005. Т. 105, № 4. С. 362-368, http://rnas .asj-oа. $\mathrm{am} / 1428$. 
18. Kaplunov J. D., Prikazchikov D. A., Rogerson G. A. On three-dimensional edge waves in semi-infinite isotropic plates subject to mixed face boundary conditions // J. Acoust. Soc. Am., 2005. vol. 118, no. 5. pp. 2975-2983. doi: 10.1121/1.2062487.

19. Zernov V., Kaplunov J. Three-dimensional edge waves in plates // Proc. R. Soc. Lond. A, 2008. vol. 464. pp. 301-318. doi : 10.1098/rspa.2007.0159.

20. Вильде М. В., Каплунов Ю. Д., Коссович Л. Ю. Краевые и интерфейсные резонансные явления в упругих телах. М.: Физматлит, 2010. 280 с.

21. Головчан В. Т., Кубенко В. Д., Шульга Н. А., Гузь А. Н., Гринченко В. Т. Пространственные задачи теории упругости и пластичности / Динамика упругих тел. Т. 5. Киев: Наук. думка, 1986. 288 с.

Поступила в редакцию $07 / \mathrm{XI} / 2014$;

в окончательном варианте - 01/XII/2014;

принята в печать - 11/XII/2014.

Vestn. Samar. Gos. Techn. Un-ta. Ser. Fiz.-mat. nauki

[J. Samara State Tech. Univ., Ser. Phys. \& Math. Sci.] 2014. Issue 4 (37). Pp. 53-64

ISSN: 2310-7081 (online), 1991-8615 (print) doi: http://dx.doi.org/10.14498/vsgtu1360

MSC: 74J15

\title{
THREE-DIMENSIONAL SURFACE WAVE IN HALF-SPACE AND EDGE WAVES IN PLATES WITH MIXED BOUNDARY CONDITIONS ON THE FRONT EDGE
}

\author{
R. V. Ardazishvili, M. V. Wilde, L. Yu. Kossovich \\ N. G. Chernyshevsky Saratov State University (National Research University), \\ 83, Astrakhanskaya st., Saratov, 410012, Russian Federation.
}

\begin{abstract}
In the first part of this paper the propagation of sinusoidal three-dimensional surface waves is investigated for an isotropic elastic half-space with mixed boundary conditions. It is assumed that the boundary is fixed in one of the tangential directions and traction free in the other directions. The exact dispersion relation is derived which shows the existence and uniqueness of the three-dimensional surface wave. The speed of this wave depends on the angle of propagation and lies between the shear wave speed and Rayleigh wave speed. The graphs of this dependence are presented for various values of Poisson ratio. In the second part of this paper the three-dimensional edge
\end{abstract}

(C) 2014 Samara State Technical University.

How to cite Reference

Ardazishvili R. V., Wilde M. V., Kossovich L. Yu. Three-dimensional surface wave in half-space and edge waves in plates with mixed boundary conditions on the front edge, Vestn. Samar. Gos. Tekhn. Univ., Ser. Fiz.-Mat. Nauki [J. Samara State Tech. Univ., Ser. Phys. \& Math. Sci.], 2014, no. 4(37), pp. 53-64. doi: 10.14498/vsgtu1360. (In Russian)

Authors Details

Roman V. Ardazishvili (ardazishvili.roman@yandex.ru), Postgraduate Student, Dept. of Mathematical Theory of Elasticity and Biomechanics.

Maria V. Wilde (Dr. Phys. \& Math. Sci.; mv_wilde@mail.ru; Corresponding Author), Professor, Dept. of Mathematical Theory of Elasticity and Biomechanics.

Leonid Yu. Kossovich (Dr. Phys. \& Math. Sci. president@sgu.ru), Professor, Head of Department, Dept. of Mathematical Theory of Elasticity and Biomechanics. 
waves in plates with mixed boundary conditions on the edge are investigated. The faces of the plate are assumed to be traction free. Both symmetric and antisymmetric solutions of three-dimensional dynamic equations of elasticity are considered. It is assumed that the edge is fixed in one of the tangential directions and traction free in the normal and the other tangential direction. Asymptotic analysis is performed, which shows that there is an infinite spectrum of higher order edge waves in such plates. The results of numerical calculations based on the modal expansion method are presented to confirm asymptotic analysis. In addition, by the numerical investigation the fundamental edge wave was found in the symmetric case (the edge is fixed in the tangential direction transversally to the faces). The phase velocity of this wave tends to some limit value depending on the Poisson ratio as the wave number increases. In the antisymmetric case the first higher order wave has the same limit value. The dispersion curves are presented for various values of Poisson ratio.

Keywords: surface waves, edge waves, Rayleigh wave, shear wave, asymptotic methods.

doi: http://dx.doi.org/10.14498/vsgtu1360

\section{ORCID}

Roman V. Ardazishvili: http://orcid.org/0000-0002-0993-805X

Maria V. Wilde: http://orcid.org/0000-0001-8198-3368

Leonid Yu. Kossovich: http://orcid.org/0000-0002-4775-7348

\section{REFERENCES}

1. Rayleigh J. On waves propagated along the surface of an elastic solid, Proc. Lond. Math. Soc., 1885, vol.s1-17, no. 1, pp. 4-11. doi: 10.1112/plms/s1-17.1.4.

2. Viktorov I. A. Types of acoustic surface waves in solids (review), Sov. Phys. Acoust., 1979, vol. 25, no. 1, pp. 1-9.

3. Destrade M., Scott N. H. Surface waves in a deformed isotropic hyperelastic material subject to an isotropic internal constraint, Wave Motion, 2004, vol.40, no. 4, pp. 347-357. doi: 10. 1016/j . wavemoti.2003.09.003.

4. Dai H. H., Kaplunov J., Prikazchikov D. A. A long-wave model for the surface elastic wave in a coated half-space, Proc. R. Soc. A, 2010, vol. 466, pp. 3097-3116. doi: 10.1098/rspa. 2010.0125.

5. Eduardo Godoy, Mario Durán, Jean-Claude Nédélec On the existence of surface waves in an elastic half-space with impedance boundary conditions, Wave Motion, 2012, vol.49, no. 6, pp. 585-594. doi: 10.1016/j. wavemoti.2012.03.005.

6. Stan Chiriţă, Michele Ciarletta, Vincenzo Tibullo Rayleigh Surface Waves on a Kelvin-Voigt Viscoelastic Half-Space, Journal of Elasticity, 2013, vol. 115, no. 1, pp. 61-76. doi: 10.1007/ s10659-013-9447-0.

7. Inder Singh Gupta Propagation of Rayleigh Waves in a Prestressed Layer over a Prestressed Halfspace, Frontiers in Geotechnical Engineering (FGE), 2013, vol.2, no. 1, pp. 16-22, https://archive.org/details/FGE009.

8. Baljeet Singh Propagation of Rayleigh Wave in a Thermoelastic Solid Half-Space with Microtemperatures, International Journal of Geophysics, 2014, vol. 2014, pp. 1-6. doi: 10. $1155 / 2014 / 474502$.

9. Konenkov Y. K. A Rayleigh-type flexural wave, Soviet Acoust. Physics, 1960, vol.6, no. 1, pp. $122-123$.

10. Belubekian M. V., Gulgazaryan G. R., Saakian A. V. The waves of rayleighs type in semi-infinite clossed circuliar cilindrical shells, Izv. NAN Armenii, Mekhanika [Mechanics. Proceedings of National Academy of Sciences of Armenia], 1997, vol. 50, no. 3-4, pp. 49-55 (In Russian), http://mechanics.asj-oa.am/180. 
11. Kaplunov J. D., Kossovich L. Yu., Wilde M. V. Free localized vibrations of a semi-infinite cylindrical shell, J. Acoust. Soc. Am., 2000, vol. 107, no. 3, pp. 1383-1393. doi: 10.1121/1. 428426.

12. Kaplunov J. D., Wilde M. V. Edge and interfacial vibrations in elastic shells of revolution, ZAMP, 2000, vol.51, no. 4, pp. 530-549. doi: 10.1007/s000330050015.

13. Fu Y. B., Brookes D. W. Edge waves in asymmetrically laminated plates, Journal of the Mechanics and Physics of Solids, 2006, vol.54, no. 1, pp. 1-21. doi: 10.1016/j.jmps. 2005. 08.007.

14. Piliposian G. T., Belubekyan M. V., Ghazaryan K. B. Localized bending waves in a transversely isotropic plate, Journal of Sound and Vibration, 2010, vol.329, no. 17, pp. 3596-3605. doi: 10.1016/j.jsv.2010.03.019.

15. Krushynska A. A. Flexural edge waves in semi-infinite elastic plates, Journal of Sound and Vibration, 2011, vol. 330, no. 9, pp. 1964-1976. doi: 10.1016/j.jsv.2010.11.002.

16. Fu Y. B., Kaplunov J. Analysis of localized edge vibrations of cylindrical shells using the Stroh formalism, Math. Mech. Solids, 2012, vol.17, no. 1, pp. 59-66. doi: 10.1177/ 1081286511412442.

17. Belubekyan V. M., Belubekyan M. V. Three-dimensional Problem of Reyleight Wave Propagation., Dokl. NAN Armenii, 2005, vol. 105, no.4, pp. 362-368 (In Russian), http: //rnas.asj-oa.am/1428.

18. Kaplunov J. D., Prikazchikov D. A., Rogerson G. A. On three-dimensional edge waves in semi-infinite isotropic plates subject to mixed face boundary conditions, J. Acoust. Soc. Am., 2005, vol.118, no. 5, pp. 2975-2983. doi: 10.1121/1.2062487.

19. Zernov V., Kaplunov J. Three-dimensional edge waves in plates, Proc. R. Soc. Lond. A, 2008, vol. 464, pp. 301-318. doi: 10.1098/rspa.2007.0159.

20. Wilde M. V., Kaplunov Yu. D., Kossovich L. Yu. Kraevye i interfeisnye rezonansnye iavleniia $v$ uprugikh telakh [Edge and interfacial resonance phenomena in elastic bodies]. Moscow, Fizmatlit, 2010, 280 pp.

21. Golovchan V. T., Kubenko V. D., Shul'ga N. A., Guz' A. N., Grinchenko V. T. Threedimensional problems of the theory of elasticity and plasticity, Dinamika uprugikh tel [Dynamics of elastic bodies], vol. 5. Kiev, Nauk. dumka, 1986, 288 pp.

Received 07/XI/2014;

received in revised form $01 / \mathrm{XII} / 2014$;

accepted 11/XII/2014. 